\title{
CLINICAL COMPARISON OF THREE TECHNIQUES FOR EVALUATING VISUAL FUNCTION BEHIND CATARACT
}

\author{
BRENDAN T. BARRETT ${ }^{1}$, PETER A. DAVISON ${ }^{2}$ and PETER EUSTACE ${ }^{3}$ \\ Glasgow and Dublin, Ireland
}

\begin{abstract}
SUMMARY
The aim of the study was to compare the ability of displacement threshold hyperacuity to identify reduced retinal/neural function in cataract patients with that of the potential acuity meter (PAM) and laser interferometer. Fifty-two patients (mean age $72.0 \pm 10.8$ years) admitted for extracapsular cataract extraction were randomly selected for inclusion in the study. Patients underwent routine refraction and $\operatorname{logMAR}$ acuity measurement on the eve of cataract surgery. Following pupillary dilation and in random order, the oscillatory displacement threshold (ODT; defined as the smallest detectable displacement of an object) was determined, and predictions of the post-operative acuity were obtained using the PAM and Rodenstock Retinometer (RR). Patients were re-examined approximately 7 weeks post-operatively when $\log M A R$ acuities and ODTs were remeasured. On the basis of the results of an independent fundus examination, patients were assigned to either a normal or a pathology group. Increasing cataract density adversely affected the predictions provided by the PAM $(p=0.0001)$ and ODT $(p=0.0001)$ techniques, but not the RR $(p=$ 0.137). One-factor ANOVA revealed that pre-operative ODT $(p=0.0001)$, PAM $(p=0.0001)$ and RR $(p=$ 0.0004) measures successfully distinguished patients with retinal/neural pathology from normals. Preoperative cut-off levels to distinguish normal from pathology cases were retrospectively determined for each technique. The cut-off values for the PAM and ODT techniques yielded higher sensitivity and specificity values than was the case for the RR. The results suggest that the predictive abilities of the PAM and

From: ${ }^{1}$ Department of Vision Sciences, Glasgow Caledonian University, Glasgow, UK; ${ }^{2}$ Department of Optometry, Dublin Institute of Technology, Dublin, Ireland; ${ }^{3}$ Department of Ophthalmology, University College Dublin, Belfield, Dublin, Ireland.

Correspondence to: Dr B. T. Barrett, Department of Vision Sciences, Glasgow Caledonian University, Cowcaddens Road, Glasgow G4 0BA, UK. Fax: (44)-41-331 3387.
\end{abstract}

ODT techniques are similar, and superior to that of the RR. Further work involving larger numbers of patients with posterior segment pathology is necessary to reveal which technique provides the best means of assessing retinal/neural function in the presence of ocular media opacities.

The improvement in visual function following cataract extraction is critically dependent upon the integrity of the retina and its neural pathways. Posterior segment disorders coincident with the cataract may grossly compromise the post-surgical visual result. It is important, therefore, to distinguish between the optical and retinal/neural components of visual loss in pre-operative cataract patients. ${ }^{1-3}$ While the presence of posterior pole dysfunction does not preclude improvement in visual performance following cataract extraction, techniques which are sensitive to reduced retinal/neural function behind cataract provide additional information with which the clinician can assess the likely benefits of surgical intervention.

The techniques currently used to evaluate neural (including retinal) function behind cataract can be divided into three groups, depending upon the approach taken to the problem. Techniques in the first group do not require the optical system of the eye to form a clear retinal image. The aim, therefore, is to provide a task which can be performed equally well irrespective of the retinal image quality. This group includes the electrophysiological tests ${ }^{4}, 5$ and the entoptic tests. ${ }^{5,6}$ An alternative approach involves the use of Maxwellian view optical systems ${ }^{7}$ to 'project' an acuity target through an optically clear portion of the cataractous lens. This approach is adopted in the design of the potential acuity meter (PAM, Mentor O\&O Inc., Hingham, MA, USA). ${ }^{8}$ The third group is comprised of the various clinical interferometers. These instruments 
also employ Maxwellian view optics ${ }^{7}$ in order to form the retinal image independently of the optical system of the eye. ${ }^{1,9-11}$ The predictive ability of techniques in each group has been widely investigated. In addition, several studies have compared the ability of techniques to identify reduced neural function in non-cataractous ${ }^{12,13}$ and cataractous eyes., ${ }^{2,14-16}$ Despite the considerable research attention which this problem has received, however, the clinical usefulness of many techniques remains controversial, ${ }^{17,18}$ and there is little consensus of opinion regarding which technique provides the best means of assessing neural integrity behind cataract.

The factors influencing the predictive ability of a particular technique have recently been discussed. ${ }^{19}$ Differing conclusions regarding predictive value may, for example, arise from differences in the prevalence of neural dysfunction in the patient sample. When two or more techniques are compared, relative predictive values are heavily influenced by the index used to gauge the success, or otherwise, of the post-operative visual result. Snellen acuity has traditionally been used to assess postoperative visual quality, and hence is typically the measure of visual function which techniques aim preoperatively to predict. When comparing techniques such as the PAM and blue field entoptoscopy, it is inappropriate to use visual acuity as the sole means of assessing the post-operative visual outcome, since the acuity predictions provided by the PAM are always likely to appear better than the entoptoscopy predictions when the gold standard for post-operative visual quality is itself visual acuity.

Rather than comparing the ability to predict the post-operative visual acuity, a more appropriate approach would be to compare the abilities of the various techniques to identify pre-operatively the presence of posterior pole dysfunction which may in turn, of course, affect the post-operative visual acuity. Future studies adopting this approach will enable fair comparison of the predictive abilities of the various techniques available, and will therefore, lead to a resolution of the issue of which technique provides the best means of assessing neural integrity in cataract patients.

One of the more recent additions to the list of techniques for evaluating neural function behind cataract involves the measurement of certain hyperacuity thresholds. Hyperacuity tasks require the observer to perceive a difference in relative spatial localisation of two or more visual stimuli. ${ }^{20}$ Under ideal conditions, thresholds may be smaller than the diameter of a foveal cone. Early efforts to use hyperacuity for the evaluation of neural function in eyes with cataract employed vernier acuity. ${ }^{21-24}$ Vernier acuity may not, however, represent the best choice of hyperacuity for the evaluation of neural integrity in cataract patients. Thresholds need to be determined for a range of separations of the stimulus features. In patients with known or suspected posterior pole pathology, thresholds need to be established for various retinal locations. A single measure of vernier acuity, therefore, provides the clinician with very little useful information. An alternative form of hyperacuity suggested for this purpose involves the measurement of oscillatory displacement thresholds (ODTs). The ODT is defined as the smallest detectable displacement of a target relative to stationary references. ${ }^{3}$ ODTs have been shown to be robust to optical image degradation as produced by simulated ${ }^{25,26}$ and real catar$\mathrm{act}^{27,28}$ and by posterior capsular opacification. ${ }^{29}$ In addition, ODTs are sensitive to the presence of posterior pole dysfunction, ${ }^{3,13}$ including amblyopia. ${ }^{30}$ Despite considerable promise, however, the clinical usefulness of ODT measurement in pre-operative cataract patients has not been validated against existing techniques.

The ability of ODTs to identify reduced neural function in eyes with clear media has recently been compared with that of the PAM and Rodenstock Retinometer (RR, Rodenstock, London, UK). ${ }^{13}$ The aim of the present study was to compare the abilities of these three techniques to identify reduced neural function in patients with cataract.

\section{METHODS}

\section{Patients}

Fifty-two patients (mean age $72.0 \pm 10.8$ years) admitted to the Mater Misericordiae Hospital in Dublin for extra-capsular cataract extraction participated in the study. Patients were examined on the eve of surgery. This consisted of refraction and visual acuity measurement. Following pupillary dilation, and in random order, the patient's ODT was measured, and retinal acuities were measured with the PAM and RR. Testing was carried out without knowledge of the patient's ocular or general medical history, past or current. Informed consent was obtained from all patients prior to testing.

Patients were seen again between 6 and 8 weeks post-operatively when, following refraction and visual acuity measurement, ODTs were remeasured. The PAM and RR were not used postoperatively for reasons unconnected with this study. On the basis of an independent ophthalmological examination carried out post-operatively, patients were allocated to a normal or a pathology group. The pathology group included the following clinical conditions: non-exudative age-related maculopathy $(n=4)$, diabetic retinopathy $(n=1)$ and amblyopia $(n=1)$. No patient in the normal $(n=46)$ or pathology $(n=6)$ group had post-operative visual 
performance reduced as a result of a complication of the surgical procedure.

\section{Acuity Measurement}

Acuities were measured using logMAR charts. ${ }^{31}$ The charts were viewed from a distance of $3.2 \mathrm{~m}$. When no letter could be seen, the viewing distance was reduced to $2.5 \mathrm{~m}$. Further reductions were used as necessary. Appropriate working distance lenses were incorporated to account for whatever distance was used and the $\operatorname{logMAR}$ acuity was determined accordingly. Following pupillary dilation, the acuity was remeasured using a $1 \mathrm{~mm}$ pinhole placed in a trial frame. The pre-operative acuity was taken as the best level of acuity exhibited by the patient, i.e. with or without the use of the pinhole. Pre-operative acuities for the 52 patients ranged from +0.14 $\log$ MAR units (20/27.6 Snellen equivalent) to 'counting fingers' at $1 \mathrm{~m}$. The median pre-operative acuity was +0.48 logMAR units (20/60.4 Snellen equivalent).

\section{ODT Measurement}

The method of generating and controlling the stimulus for ODT measurement has been described previously. ${ }^{13}$ The stimulus was presented on a visual display terminal and consisted of three vertical white lines on a black background (Michelson contrast $97.6 \%)$. Each line subtended $12 \times 60$ arcmin when viewed from a distance of $6 \mathrm{~m}$, and the separation between the inner edges of adjacent lines, when stationary, was $33 \mathrm{arc}$ min. One of the two outer lines was made to oscillate with a square waveform (temporal frequency $2 \mathrm{~Hz}$ ). The centre line remained stationary throughout the test, and thus acted as a reference against which the movement of the line (right or left) could be judged. The patient's task was to state whether the right-hand line or lefthand line was moving. The line continued to oscillate until the patient responded, at which point the response was entered by the experimenter (B.T.B.) via the keyboard. When no movement could be detected the patient was encouraged to guess. No feedback was given regarding the accuracy of response.

Thresholds were determined using a two-up/onedown forced choice staircase procedure. ${ }^{32}$ The initial amplitude of oscillation was 150 seconds of arc. The stimulus was viewed monocularly with appropriate refractive correction in place. Threshold estimation was carried out in a dimly lit room after the patient had been allowed several minutes to adapt to the surrounding luminance levels. The procedure typically took 5-8 minutes to complete.

\section{Laser Interferometer}

The interferometer used was a Rodenstock Retin- ometer (RR). ${ }^{1,9}$ This instrument mounts on a slit lamp. An interference fringe pattern is generated when the beams of monochromatic light produced by the instrument interfere constructively and destructively on the retina. The task of the examiner is to direct these beams through clear portions (i.e. 'windows') in the cataractous lens. The patient is required to detect whether the orientation of the grating pattern is horizontal, vertical or oblique (i.e. $45^{\circ}$ or $135^{\circ}$ ). The grating with the highest spatial frequency whose orientation could be correctly identified on two successive presentations was taken as the predicted post-operative acuity. Highly ametropic patients wore refractive correction for the test.

\section{Potential Acuity Meter}

The Guyton-Minkowski Potential Acuity Meter $(\mathrm{PAM})^{8}$ is a slit lamp attachment which projects a miniature Snellen chart into the eye via a beam which narrows to a diameter of $0.1 \mathrm{~mm}$. As with the $\mathrm{RR}$, the task of the examiner is to direct this beam through a 'window' in the cataractous lens. In accordance with the manufacturer's instructions, the smallest line on which three characters could be correctly identified was taken as the predicted postoperative acuity. Astigmatic patients and patients with high ametropia wore refractive correction for the test.

\section{RESULTS}

Any technique which aims to evaluate neural function in patients with cataract must be capable of providing a pre-operative result which is independent of the presence of the cataract. The extent to which the three techniques (ODT, PAM and RR) fulfilled this requirement was examined using correlation analysis between the pre-operative

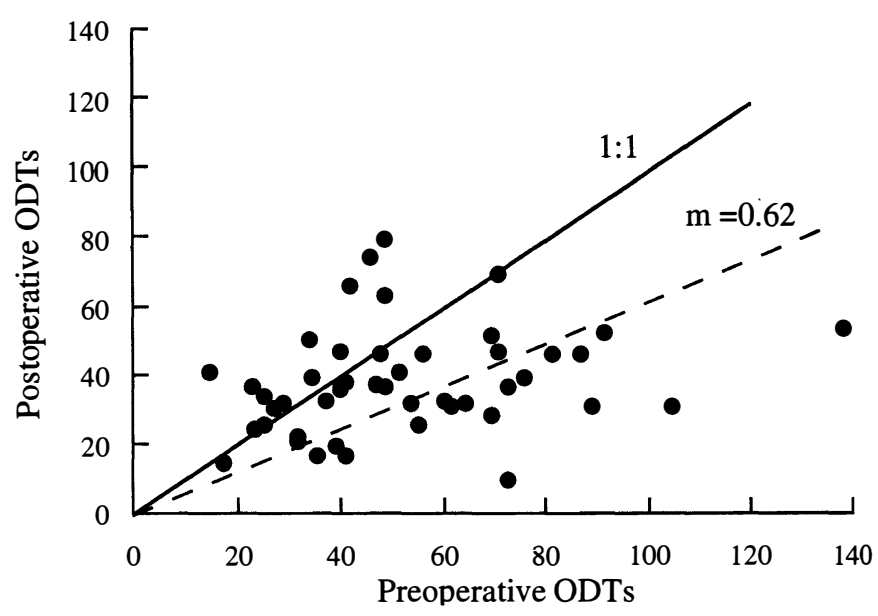

Fig. 1. Pre-operative ODTs versus post-operative ODTs for the normal group only. The majority of data points are located beneath the 1:1 line, indicating that performance was affected by the cataract. The dashed line is the regression line passing through the origin. 
Table I. Sensitivities and specificities of the three techniques for the cut-off values determined from $\mathrm{ROC}^{33}$ analysis

\begin{tabular}{lcc}
\hline & Sensitivity (\%) & Specificity (\%) \\
\hline ODT & 100 & 98 \\
PAM & 100 & 91 \\
RR & 83 & 72 \\
\hline
\end{tabular}

measures obtained using the three techniques and the pre-operative $\log$ MAR acuities for the patients assigned to the normal group. Pre-operative logMAR acuities were significantly correlated with preoperative ODTs $\left(r^{2}=0.29, p=0.0001\right)$ and with preoperative predictions of the post-operative acuity obtained using the PAM $\left(r^{2}=0.41, p=0.0001\right)$. Predictions obtained using the RR were not, however, significantly correlated with pre-operative $\log$ MAR acuities $\left(r^{2}=0.05, p=0.137\right)$. Since preoperative acuity was limited in patients by optical factors alone, it is clear that the RR was the only technique on which pre-operative performance was truly independent of the presence of cataract. The effects of cataract upon ODTs may be further examined in Fig. 1 which shows pre-operative thresholds plotted against post-operative thresholds for patients assigned to the normal group. Clearly, if ODTs were unaffected by cataract, pre- and postoperative thresholds would be identical. The slope of the regression line passing through the origin is only 0.62 , thus confirming that lower thresholds were measured post-operatively. Pre- and post-operative ODTs were, however, significantly correlated for the combined data of the normal and pathology groups, consistent with the view that ODTs demonstrate considerable resistance to optical image degradation $\left(r^{2}=0.49, p=0.0001\right)$.

The predictive value of a given technique is determined by its ability to distinguish patients with

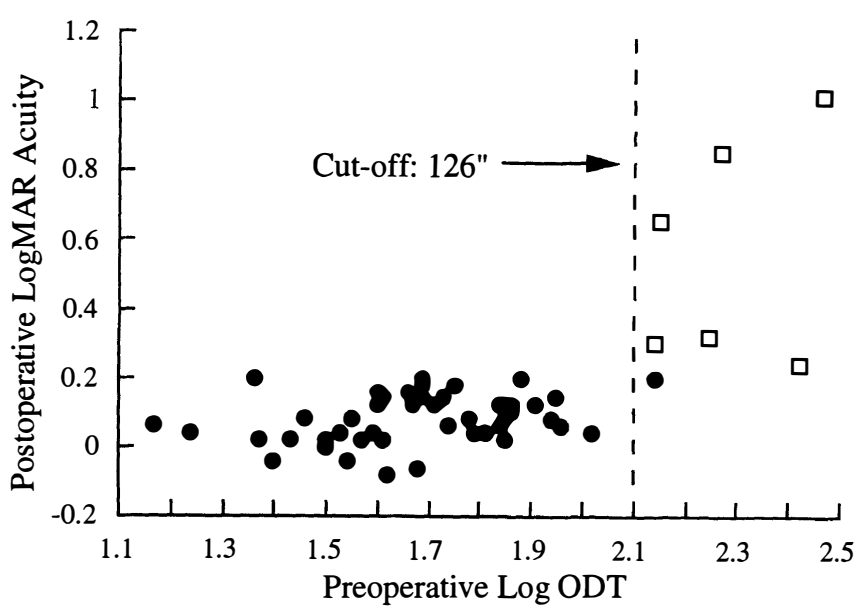

Fig. 2. Pre-operative ODTs versus post-operative $\log M A R$ scores for the normal (filled circles) and pathology (open squares) groups. The dashed line represents the cut-off value determined from ROC analysis. ${ }^{33}$ Six of the seven patients $(86 \%)$ with ODTs above this cut-off had reduced neural function.

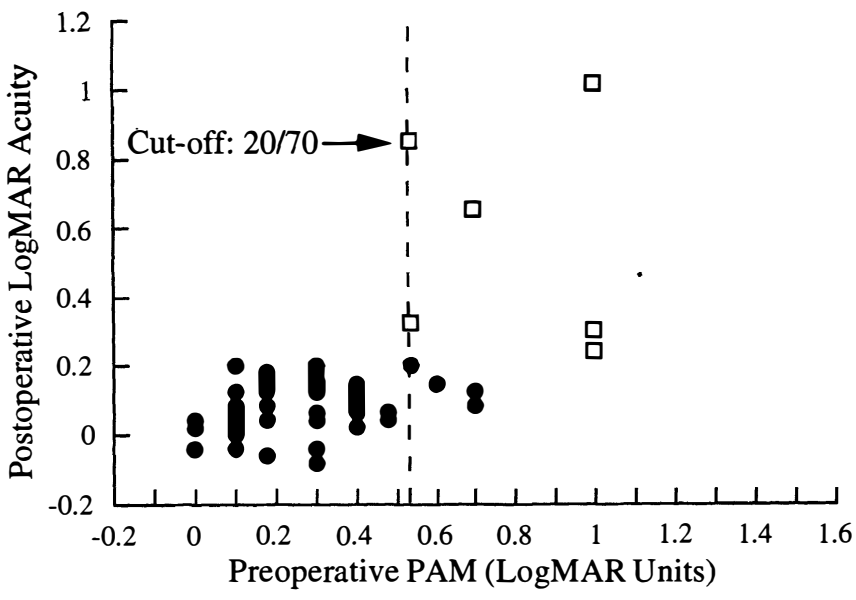

Fig. 3. Predictions of post-operative acuity obtained using the PAM (in logMAR units) plotted against the actual postoperative $\log M A R$ acuities for patients in the normal (filled circles) and pathology groups (open squares) groups. The dashed line represents the cut-off value determined from ROC analysis.

posterior segment dysfunction from those with normal neural function. A one-factor ANOVA revealed that pre-operative ODTs were significantly higher in patients with neural disease than in normals $\left(F_{1,50}=48.35, p=0.0001\right)$. The PAM $\left(F_{1,50}=47.21, p\right.$ $=0.001)$ and $\operatorname{RR}\left(F_{1,49}=14.18, p=0.0004\right)$ were also successful in distinguishing normal patients from those with neural dysfunction.

To decide upon a cut-off value above which preoperative performance could be considered to be indicative of reduced neural function, receiveroperating characteristic (ROC) analysis ${ }^{33}$ was applied to the data for the three techniques. In the case of ODT measurement, this analysis revealed that the predictive ability is maximal for a preoperative ODT value of $2.10 \log$ units or 126 seconds

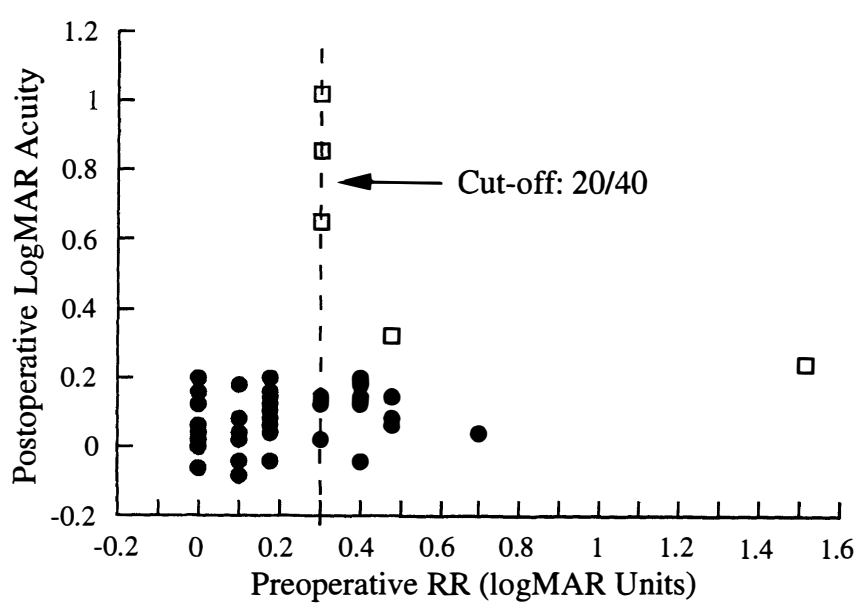

Fig. 4. Predictions of post-operative acuity obtained using the $R R$ (in $\log M A R$ units) plotted against the actual postoperative $\log M A R$ acuities for the normal (filled circles) and pathology groups (open squares). Note that this cut-off (dashed line) is less successful in discriminating between the two groups. 
of arc. If the cut-off is increased or decreased, a trade-off occurs between improvements in sensitivity and specificity. The ROC analysis for the other techniques yielded cut-off values of $0.54 \log$ MAR units (Snellen equivalent 20/70) and 0.30 logMAR units (Snellen equivalent 20/40) for the PAM and RR respectively. Table I shows sensitivity and specificity values for each technique determined using these cut-off values. On the basis of these preliminary results, the predictive ability of the PAM and ODT measurements appear superior to that of the RR.

Figs. 2-4 show pre-operative performance for the three techniques (ODT, PAM and RR respectively) plotted against post-operative logMAR acuity for the normal and pathology groups. Also shown are the cut-off values determined in each case from ROC analysis. It is clear that the cut-off values for the ODT (Fig. 2) and PAM (Fig. 3) techniques differentiated between the two groups with greater success than was the case for the RR (Fig. 4).

\section{DISCUSSION}

The ability of a technique to assess neural integrity behind cataract is determined by the extent to which the following criteria are fulfilled: (1) the independence of the pre-operative result from the presence of cataract, and (2) the sensitivity of the result to the conditions which will impair post-operative visual performance.

The results of the present study suggest that the RR fulfils the first criterion to a greater extent than the PAM or ODT techniques. Faulkner ${ }^{9}$ reported superior penetration of media opacities using interferometric techniques by comparison with the PAM. Despite this superior penetration, several studies have reported that, in common with PAM predictions, interferometric acuity predictions become less accurate in eyes with dense cataracts. ${ }^{15,16,34}$ ODTs have been shown to demonstrate considerable resistance to optical image degradation, ${ }^{25,27}$ though no previous study has compared this resistance with that of other techniques. In common with two preand post-operative studies, ${ }^{3,26}$ the present results support the view that this resistance breaks down when eyes with more dense cataracts are examined. However, while poorer than the RR, ODTs appear to demonstrate greater resistance to optical image degradation than the PAM.

Pre-operative performance on all three techniques was significantly poorer for the pathology group than for the normal patients. This indicates that each technique is sensitive to reduced neural function. All three techniques appear, therefore, to fulfil the second pre-requisite outlined above, though to a lesser extent in the case of the RR. The PAM has previously been shown to demonstrate greater sensitivity to neural deficits by comparison with interferometric techniques in non-cataractous eyes, ${ }^{12,13}$ though in cataractous eyes conflicting reports exist with regard to the relative predictive value of these techniques. The sensitivity of ODTs to posterior segment disorders, including amblyopia in eyes with clear media ${ }^{3,13,30}$ and in cataractous eyes, ${ }^{3,26}$ has also been described.

The results of the current study suggest that both the PAM and ODT techniques offer a more effective means of identifying patients with coincident posterior segment pathology than that provided by the RR (Table I). Whilst the retinal acuities measured with the RR demonstrated greater independence of the presence of cataract than the pre-operative measures obtained using the other techniques, this instrument performed poorly in patients with reduced neural function. Since the RR is used to predict the postoperative visual acuity, it is reasonable to consider predictive failures in terms of discrepancies between predicted acuity and the acuity actually achieved. The RR overestimated the post-operative acuity in three of the four patients with age-related maculopathy, and grossly underestimated the acuity in the fourth (Fig. 4). In addition, one of the patients in the pathology group failed to see even the coarsest interference fringe pattern. Poor agreement between interferometric and letter acuities in eyes with nonexudative age-related maculopathy has previously been documented. ${ }^{13,35}$ While the results suggest that the predictive ability of the RR is poorer than that of the other techniques, it appears that the predictive abilities of the PAM and ODT are approximately equal (Table I).

In considering the results of the current study, cognisance must be taken of the small number of pathology cases in the patient sample. Future studies involving larger patient numbers will reveal whether the relative predictive values of the techniques were influenced by the small size of the pathology group. Furthermore, these studies will enable the predictive ability of the proposed cut-off values determined for the three techniques to be assessed. It is interesting to note, however, that the proposed ODT cut-off level of 126 seconds of arc agrees well with the value of 102 seconds of arc recently proposed by McGraw et al. ${ }^{29}$ who investigated the ability of this technique to identify patients with reduced neural function prior to Nd:YAG laser capsulotomy.

In conclusion, the results of this study suggest that the predictive abilities of the PAM and ODT techniques in patients with cataract are similar. The RR appears less well able pre-operatively to distinguish patients with posterior segment dysfunction from normals.

Key words: Cataract, Hyperacuity, Interferometer, Potential acuity meter, Retinal/neural function. 


\section{REFERENCES}

1. Faulkner W. Laser interferometric prediction of postoperative visual acuity in patients with cataracts. Am J Ophthalmol 1983;95:626-36.

2. Spurny RC, Zaldivar R, Belcher CD, Simmons RJ. Instruments for predicting visual acuity: a clinical comparison. Arch Ophthalmol 1986;104:196-200.

3. Whitaker D, Deady J. Prediction of visual function behind cataract using displacement threshold hyperacuity. Ophthalmic Physiol Opt 1989;9:20-4.

4. Brodie SE. Evaluation of cataractous eyes with opaque media. Int Ophthalmol Clin 1987;27:153-62.

5. Skalka HW. Blue field entoptoscopy and VER in preoperative cataract evaluation. Ophthalmic Surg 1981;12:642-5.

6. Sinclair SH, Loebl M, Riva CE. Blue field entoptic phenomenon in cataract patients. Arch Ophthalmol 1979;97:1092-5.

7. Westheimer G. The maxwellian view. Vision Res 1966;6:669-89.

8. Minkowski JS, Palese M, Guyton DL. Potential acuity meter using a minute pinhole aerial aperture. Ophthalmology 1983;90:1360-8.

9. Faulkner W. Predicting acuities in capsulotomy patients: interferometers and Potential Acuity Meter. J Am Intraocul Implant Soc 1983;9:434-7.

10. Guyton DL. Instruments for measuring visual acuity behind cataracts. Ophthalmology 1982;89:34-9.

11. Lotmar W. Apparatus for the measurement of retinal visual acuity by moiré fringes. Invest Ophthalmol Vis Sci 1980;19:393-400.

12. Fish GE, Birch DG, Fuller DG, Straach R. A comparison of visual function tests in eyes with maculopathy. Ophthalmology 1986;93:1177-82.

13. Barrett BT, Davison PA, Eustace PE. The effects of posterior segment disorders on oscillatory displacement thresholds, and on acuities as measured using the Potential Acuity Meter and laser interferometer. Ophthalmic Physiol Opt 1994;14:132-8.

14. Davis ET, Sherman J, Bass SJ, Schnider CM. Presurgical prediction of post-surgical visual function in cataract patients: multivariate statistical analyses of test measures. Clin Vis Sci 1991;6:191-207.

15. Minkowski JS, Guyton DL. New methods for predicting visual acuity after cataract surgery. Ann Ophthalmol 1984;16:511-6.

16. Datiles MB, Edwards PA, Kaiser-Kupfer MI, McCain L, Podgor M. A comparative study between the PAM and laser interferometer in cataracts. Graefes Arch Clin Exp Ophthalmol 1987;225:457-60.

17. Halliday BL, Ross JE. Predicting acuities in patients with cataract. Br J Ophthalmol 1983;67:787.

18. Faulkner W. Predicting acuities in patients with cataract. Br J Ophthalmol 1983;67:786-7.

19. Barrett BT. Comparing techniques for evaluating retinal/neural function behind cataract. Ophthalmic Physiol Opt 1994;14:433-4.

20. Westheimer G. Visual acuity and hyperacuity. Invest Ophthalmol Vis Sci 1975;14:570-2.

21. Enoch JM, Williams RA, Essock EA, Fendick M. Hyperacuity: a promising means of evaluating vision through cataract. In: Osborne NN, Chader GJ, editors. Progress in retinal research, vol 4. Oxford: Pergamon Press, 1985:67-88.

22. Essock EA, Williams RA, Enoch JM, Raphael S. The effects of image degradation by cataract on vernier acuity. Invest Ophthalmol Vis Sci 1984;25:1043-50.

23. Enoch JM, Williams RA, Essock EA, Barricks M. Hyperacuity perimetry: assessment of macular function through ocular opacities. Arch Ophthalmol 1984;102:1164-8.

24. Essock EA, Enoch JM, Williams RA, Barricks M, Raphael S. Joint application of hyperacuity perimetry and gap tests to assess visual function behind cataracts: initial trials. Doc Ophthalmol 1985;60:293-312.

25. Whitaker D, Buckingham T. Oscillatory movement displacement thresholds: resistance to optical image degradation. Ophthalmic Physiol Opt 1987;7:121-5.

26. Barrett BT, Davison PA, Eustace PE. Assessing retinal/neural function in patients with cataract using oscillatory displacement thresholds. Optom Vis Sci 1994;71:801-8.

27. Whitaker D, Elliott DB. Towards establishing a clinical displacement threshold technique to evaluate visual function behind cataract. Clin Vis Sci 1989;4:61-9.

28. Bueno G, Hurst MA. Displacement threshold hyperacuity as a predictor of postsurgical visual performance in patients with cataract. Invest Ophthalmol Vis Sci 1995;36:686-91

29. McGraw PV, Brosnahan D, Winn B, Whitaker D. Assessment of retinal-neural function before neodymium:YAG laser capsulotomy. Invest Ophthalmol Vis Sci 1995;36:1155-62.

30. Buckingham T, Watkins R, Bansal $\mathrm{P}$, Bamford $\mathrm{K}$. Hyperacuity thresholds for oscillatory movement are abnormal in strabismic and anisometropic amblyopes. Optom Vis Sci 1991;68:351-6.

31. Ferris FL, Kassoff A, Bresnick GH, Bailey I. New visual acuity charts for clinical research. Am J Ophthalmol 1982;94:91-6.

32. Wetherill GB, Levitt H. Sequential estimation of points on the psychometric function. Br J Math Stat Psychol 1965;18:1-10.

33. Green DM, Swets JA. Signal detection theory and psychophysics. New York: Wiley, 1966:5-53.

34. Christenbury JD, McPherson SD. Potential Acuity Meter for predicting postoperative visual acuity in cataract patients. Am J Ophthalmol 1985;99:365-6.

35. Bloom TD, Fishman GA, Traubert BS. Laser interferometric visual acuity in senile macular degeneration. Arch Ophthalmol 1983;101:925-6. 